\title{
On the Irregularity Characterization of Mean Graphs
}

\section{Tamás Réti, István Barányi}

Bánki Donát Faculty of Mechanical and Safety Engineering, Óbuda University, Népszínház u. 8, H-1081 Budapest, Hungary, reti.tamas@bgk.uni-obuda.hu, baranyi.istvan@bgk.uni-obuda.hu

\begin{abstract}
A connected non-regular graph $G$ with $n$ vertices and $m$ edges is called a mean graph, if there exists a $p \geq 2$ integer for which $p=[G]=2 \mathrm{~m} / \mathrm{n}$ holds. The topological index $p=p(G)$ is called the centrality parameter of graph $G$. It is obvious that, if $G$ is a mean graph, then its centrality parameter $p(G)$ is a uniquely defined positive integer. Mean graphs represent a particular subset of connected non-regular graphs. In this note, by presenting relevant examples, some structural irregularity properties of mean graphs are studied and characterized. Comparing the degree deviations $S(G)$ and $S(H)$ of mean graphs $G$ and $H$ having equal centrality parameter $p(G)=p(H)$ it is proved that if the only difference in the corresponding degree sequences of $G$ and $H$ is that the number of vertices of degree $p$ is different, then $S(G)=S(H)$. The smallest mean graph is the 4-vertex unicyclic graph having a degree sequence $(3,2,2,1)$. This graph is isomorphic to the 4-vertex antiregular graph $A_{4}$, for which $S\left(A_{4}\right)=2$ holds. Using comparative tests on preselected connected graphs it has been shown that the degree deviation $S(G)$ is poorly suited for discriminating among non-regular graphs.
\end{abstract}

Keywords: non-regular graphs; irregularity indices; antiregular graphs; complete split graphs; balanced bidegreed graphs; degree deviation

\section{Preliminary}

Let $\mathrm{G}=(\mathrm{V}, \mathrm{E})$ be a finite, simple connected graph with $\mathrm{n}$ vertices and $\mathrm{m}$ edges. For connected graph $\mathrm{G}$, denote by $\mathrm{d}(\mathrm{v})$ the degree of a vertex $\mathrm{v}$ and by $\mathrm{e}=\mathrm{uv}$ the edge connecting vertices $\mathrm{u}$ and $\mathrm{v}$.

Let $\Delta=\Delta(\mathrm{G})$ and $\delta=\delta(\mathrm{G})$ be the maximum and minimum degrees, respectively, of vertices of $\mathrm{G}$, where $\mathrm{N}_{\Delta}$ and $\mathrm{N}_{\delta}$ stand for the number of of vertices of degree $\Delta=\Delta(\mathrm{G})$ and $\delta=\delta(\mathrm{G})$, respectively. The average degree of a graph $\mathrm{G}$ denoted by $[G]$, it is equal to $[\mathrm{G}]=2 \mathrm{~m} / \mathrm{n}$.

Using the standard terminology [1], a graph $\mathrm{G}$ is called R-regular if all its vertices have the same degree R. A connected graph is called non-regular if it contains at 
least two vertices with different degrees. A non-regular graph $\mathrm{G}$ is said to be $\mathrm{k}$-degreed, if its degree set contains exactly $\mathrm{k}$ different degrees. Consequently, a bidegreed graph $\mathrm{G}(\Delta, \delta)$ is a non-regular graph whose vertices have exactly two different degrees. An $n \geq 4$ vertex bidegreed graph $\mathrm{G}(\Delta, \delta)$ is called a balanced graph if $\mathrm{n}$ is an even integer for which $\mathrm{N}_{\Delta}=\mathrm{N}_{\delta}=\mathrm{n} / 2$ holds. Among $\mathrm{n}$-vertex connected bidegreed graphs, path $\mathrm{P}_{4}$ with degree sequence $(2,2,1,1)$ and the socalled diamond graph $\mathrm{G}_{\mathrm{D}}$ with degree sequence $(3,3,2,2)$ represent the smallest balanced bidegreed graphs.

As usual, the cyclomatic number of a connected graph with $\mathrm{n}$ vertices and $\mathrm{m}$ edges is defined as $\mathrm{Q}=\mathrm{Q}(\mathrm{G})=\mathrm{m}-\mathrm{n}+1$. A connected graph $\mathrm{G}$ having $\mathrm{Q}(\mathrm{G})=\mathrm{k} \geq 1$ cycles is said to be a k-cyclic graph. As a particular case, if $Q(G)=0$ holds the corresponding acyclic graph is called a tree graph. A tree with $\mathrm{n}$ vertices has exactly $n-1$ edges.

For a connected graph $\mathrm{G}$ with $\mathrm{n}$ vertices and $\mathrm{m}$ edges, the mean degree of $\mathrm{G}$ denoted by $[\mathrm{G}]$ is defined as $[\mathrm{G}]=2 \mathrm{~m} / \mathrm{n}$. A non-regular graph $\mathrm{G}$ is called a mean graph if there exists a positive integer $\mathrm{p}$ for which $\mathrm{p}=[\mathrm{G}]$ holds. This positive integer $\mathrm{p}$ is a uniquely defined graph invariant, it is said to be the centrality parameter of graph G. From the definition of mean graphs it follows that the centrality parameter is determined by the degree sequence of graphs, the value of $\mathrm{p}(\mathrm{G})$ does not depend on the distances between vertices in a graph. Consequently, the definition of mean graphs is independent of the distance-based centrality concept known from classical graph theory.

A connected graph $\mathrm{G}$ is called a really mean graph if it is a mean graph and the degree set of $\mathrm{G}$ contains at least one vertex of degree $\mathrm{p}$. In a really mean graph a vertex of degree $\mathrm{p}$ is called a mean vertex.

The number of mean vertices in a mean graph $G$ is denoted by $N_{p}(G)$. A mean graph $G$ is said to be a minimal graph if it has no mean vertices. An edge uv in a really mean graph $\mathrm{G}$ is called a mean edge if $\mathrm{d}(\mathrm{u})=\mathrm{d}(\mathrm{v})=\mathrm{p}$ holds. The number of mean edges of $G$ is denoted by $M_{p}(G)$.

By definition, a topological invariant IT(G) of a graph $\mathrm{G}$ is called an irregularity index if $I T(G) \geq 0$ and $I T(G)=0$ if and only if graph $G$ is a regular graph. The degree deviation $\mathrm{S}(\mathrm{G})$ of a graph $\mathrm{G}$ belongs to the family of most popular graph irregularity indices. This graph invariant was introduced by Nikiforov [2], and for a connected non-regular graph $\mathrm{G}$ with $\mathrm{n}$ vertices and $\mathrm{m}$ edges it is defined as

$S(G)=\sum_{i=1}^{n}\left|d_{i}-\frac{2 m}{n}\right|=\sum_{i=1}^{n}\left|d_{i}-[G]\right|$. 


\section{Some Fundamental Properties of Mean Graphs}

It is obvious that every non-regular unicyclic graph $\mathrm{G}$ is a really mean graph with identical centrality parameter $\mathrm{p}(\mathrm{G})=2$. The smallest mean graph is the 4 -vertex unicyclic graph with a degree sequence $(3,2,2,1)$. It is isomorphic to the 4-vertex antiregular graph $\mathrm{A}_{4}$, for which $\mathrm{S}\left(\mathrm{A}_{4}\right)=2$ holds.

Proposition 1 Let $\mathrm{G}$ be a non-regular unicyclic graph and denote by $\mathrm{H}$ the unicyclic graph generated by inserting into $G$ some vertices of degree 2 . Then $\mathrm{S}(\mathrm{G})=\mathrm{S}(\mathrm{H})$.

Proof. Because the average degree $[\mathrm{G}]=2 \mathrm{~m} / \mathrm{n}$ of a unicyclic graph $\mathrm{G}$ is equal to 2 , it follows that $\mathrm{S}(\mathrm{G})$ and $\mathrm{S}(\mathrm{H})$ are independent of the number of vertices with degree 2 , consequently $\mathrm{S}(\mathrm{G})=\mathrm{S}(\mathrm{H})$ holds. This observation for 8 and 12 vertex unicyclic graphs $\mathrm{U}_{8}$ and $\mathrm{U}_{12}$ is demonstrated in Fig. 1 .
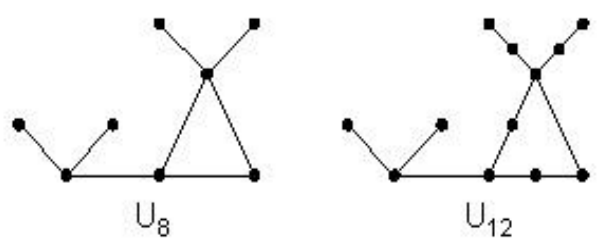

Figure 1

Four-degreed unicyclic graphs $\mathrm{U}_{8}$ and $\mathrm{U}_{12}$ with centrality parameter of $\mathrm{p}=2$

Graph $\mathrm{U}_{8}$ with 8 vertices has a degree sequence $\operatorname{DDs}\left(\mathrm{U}_{8}\right)=\left(4^{1}, 3^{2}, 2^{1}, 1^{4}\right)$ and graph $U_{12}$ with 12 vertices has a degree sequence $\operatorname{DDs}\left(U_{12}\right)=\left(4^{1}, 3^{2}, 2^{5}, 1^{4}\right)$. Because the difference between degree sequences of two graphs is represented only in the different numbers of vertices of degree 2, this implies that $\mathrm{U}_{8}$ and $\mathrm{U}_{12}$ have identical degree deviation $\mathrm{S}\left(\mathrm{U}_{8}\right)=\mathrm{S}\left(\mathrm{U}_{12}\right)=8$.

Based on the above considerations the following proposition can be obtained.

Proposition 2 Let $\mathrm{G}$ be a mean graph having $\mathrm{n}$ vertices and $\mathrm{m}$ edges and a centrality parameter $p=p(G)$. Denote by $H$ the graph constructed from $G$ by inserting into $\mathrm{G}$ finite number vertices of degree $\mathrm{p}$. Then $\mathrm{H}$ will be a really mean graph, consequently $\mathrm{p}(\mathrm{G})=\mathrm{p}(\mathrm{H})$ and $\mathrm{S}(\mathrm{G})=\mathrm{S}(\mathrm{H})$ hold. It follows that the number $\mathrm{N}_{\mathrm{p}}(\mathrm{H})$ of mean vertices of degree $\mathrm{p}$ in $\mathrm{H}$ can be arbitrarily large.

Example 1 One bidegreed and two tridegreed mean graphs are depicted in Fig. 2. These graphs have different degree sequences $\operatorname{DDs}\left(\mathrm{Y}_{6}\right)=\left(5^{3}, 3^{3}\right), \operatorname{DDs}\left(\mathrm{TY}_{7}\right)=$ $\left(5^{3}, 4^{1}, 3^{3}\right), \operatorname{DDs}\left(\mathrm{TY}_{9}\right)=\left(5^{3}, 4^{3}, 3^{3}\right)$ but identical degree deviation $\mathrm{S}\left(\mathrm{Y}_{6}\right)=\mathrm{S}\left(\mathrm{TY}_{7}\right)$ $=\mathrm{S}\left(\mathrm{TY}_{9}\right)=6$. 

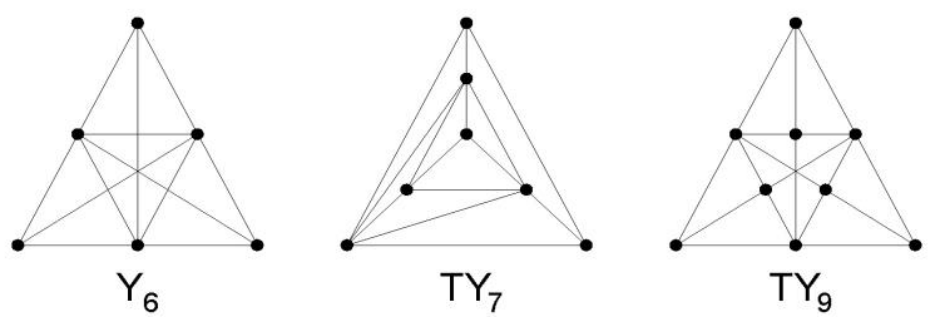

Figure 2

Graphs with identical centrality parameter $\mathrm{p}=4$

Remark 1 For an n-vertex mean graph $G$ consider the topological invariant defined by $n / p(G)$. It is conjectured that for a mean graph $G$ the sharp inequality $\mathrm{n} / \mathrm{p}(\mathrm{G}) \geq 3 / 2$ holds. For example, $\mathrm{n} / \mathrm{p}\left(\mathrm{Y}_{6}\right)=3 / 2$ for graph $\mathrm{Y}_{6}$ depicted in Fig. 2 .

Proposition 3 There are no mean graphs with cyclomatic number $\mathrm{Q}=0,2$ and 3 .

Proof. For an n-vertex connected graph G

$p(G)=\frac{2(Q(\mathrm{G})+n-1)}{n}=\frac{2 Q(\mathrm{G})}{n}+2-\frac{2}{n}$

It follows that if $\mathrm{Q}(\mathrm{G})=0,2$ or 3 , then $\mathrm{G}$ cannot be a mean graph.

Proposition 4 For the centrality parameter $\mathrm{p}$ of a mean graph the inequality $2 \leq \mathrm{p}$ $\leq \Delta-1$ holds.

Proof. As an example, consider the unicyclic graphs depicted in Fig. 3. For these mean graphs $\Delta=3, \delta=1$ and $2=\mathrm{p}=\Delta-1=2$ holds.
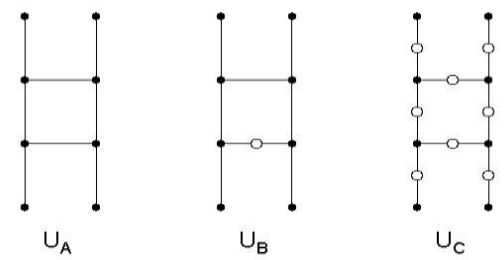

Figure 3

Tridegreed graphs $U_{B}$ and $U_{C}$ constructed from bidegreed unicyclic graph $U_{A}$

As can be seen, the number of mean vertices can be an arbitrary non-negative integer (i.e. $\mathrm{N}_{2}=0,1,2,3, \ldots$ ).

Proposition 5 Let $\mathrm{j} \geq 3$ be an arbitrary integer. Then there exists a bidegreed balanced mean graph $F_{n}$ with $n=2 j$ vertices for which $p\left(F_{n}\right)=3$ and $Q\left(F_{n}\right)=1+n / 2$ and $\mathrm{S}\left(\mathrm{F}_{\mathrm{n}}\right)=\mathrm{n}$ hold. 
Proof. Consider the infinite sequence of $n$-vertex balanced bidegreed mean graphs $\mathrm{F}_{\mathrm{n}}$ depicted in Fig. 4.

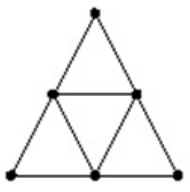

$\mathrm{F}_{6}$

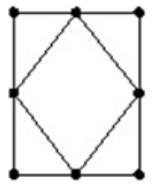

$\mathrm{F}_{8}$

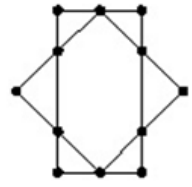

$\mathrm{F}_{12}$

Figure 4

Bidegreed balanced mean graphs $F_{n}$ having degree deviation of $S\left(F_{n}\right)=n$

The fundamental property of graphs $F_{n}$ with $n \geq 6$ vertices is that $p\left(F_{n}\right)=3, Q\left(F_{n}\right)=1$ $+n / 2$ and $S\left(F_{n}\right)=n$ hold for them. From bidegreed graphs $F_{n}$ tridegreed really mean graphs of various type can be constructed by inserting vertices of degree 3 into $F_{n}$.

Proposition 6 Let $j \geq 3$ be an arbitrary integer. Then there exists an infinite sequence of tridegreed really mean graphs $H_{n}$ with $n=2 j$ vertices for which $\mathrm{p}\left(\mathrm{H}_{\mathrm{n}}\right)=3, \mathrm{Q}\left(\mathrm{H}_{\mathrm{n}}\right)=1+\mathrm{n} / 2$ and $\mathrm{S}\left(\mathrm{H}_{\mathrm{n}}\right)=4$ hold.

Proof. Consider the tridegreed mean graphs depicted in Fig. 5.

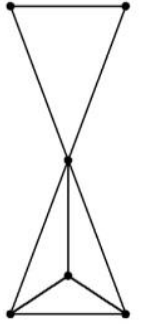

$\mathrm{H}_{6}$

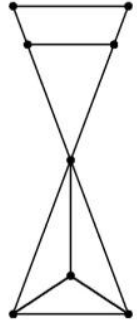

$\mathrm{H}_{8}$

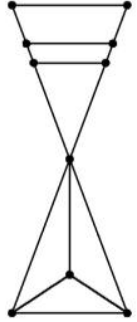

$\mathrm{H}_{10}$

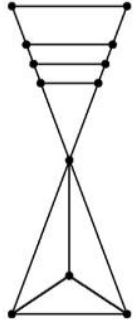

$\mathrm{H}_{12}$

Figure 5

Tridegreed mean graphs $\mathrm{H}_{\mathrm{n}}$ having identical degree deviation, $\mathrm{S}=4$

Graphs $\mathrm{H}_{6}, \mathrm{H}_{8}, \mathrm{H}_{10}$ and $\mathrm{H}_{12}$ contain 3, 5, 7 and 9 mean vertices, respectively. It is easy to check that for all tridegreed graphs $\mathrm{p}\left(\mathrm{H}_{\mathrm{n}}\right)=3, \mathrm{Q}\left(\mathrm{H}_{\mathrm{n}}\right)=1+\mathrm{n} / 2$ and $\mathrm{S}\left(\mathrm{H}_{\mathrm{n}}\right)=4$ hold.

There exist infinitely many complete bipartite graphs belonging to the family of mean graphs. It is easy to prove the following proposition.

Proposition 7 Let $\mathrm{K}_{\Delta, \delta}$ be a complete bipartite graph with $\mathrm{n}=\Delta+\delta$ vertices and $\mathrm{m}=$ $\Delta \delta$ edges where $\delta \geq 2$ even integer and $\Delta=3 \delta$. Then $\mathrm{K}_{\Delta, \delta}$ is a mean graph with a centrality parameter $\mathrm{p}\left(\mathrm{K}_{\Delta, \delta}\right)=\Delta / 2=3 \delta / 2$ and $\mathrm{S}\left(\mathrm{K}_{\Delta, \delta}\right)=\mathrm{p}\left(\mathrm{K}_{\Delta, \delta}\right)(\Delta-\delta)=\mathrm{m}$.

Remark 2 The smallest complete bipartite mean graph is the graph $\mathrm{K}_{2,6}$ with 8 vertices and 12 edges, for which $\mathrm{p}\left(\mathrm{K}_{2,6}\right)=3$ and $\mathrm{S}\left(\mathrm{K}_{2,6}\right)=\mathrm{m}=12$. 


\section{Pseudo-Antiregular Mean Graphs}

A connected n-vertex graph $A_{n}$ whose degree set consists of n-1 elements is called an antiregular graph $[3,4,5]$. It follows that a connected antiregular graph has exactly two vertices of the same degree. These two vertices with same degree are called exceptional vertices [5].

Lemma 1 [6]: Let $\mathrm{G}$ be an n-vertex connected triangle-free graph. Then for every edge $u v$ in $\mathrm{G}$ the inequality $\mathrm{d}(\mathrm{u})+\mathrm{d}(\mathrm{v}) \leq \mathrm{n}$ holds.

Lemma 2 [4, 5]: Two vertices $u$ and $v$ of a connected $n$-vertex antiregular graph $A_{n}$ are adjacent if and only if $d(u)+d(v) \geq n$.

Remark 3 There exists n-vertex connected graph $\mathrm{G}$ for which $\mathrm{d}(\mathrm{u})+\mathrm{d}(\mathrm{v}) \geq \mathrm{n}$ holds for every edge uv of $\mathrm{G}$, but $\mathrm{G}$ does not belong to the family of antiregular graphs. For example, such graphs where $d(u)+d(v)=n$ holds for every edge uv are the n-vertex stars $S_{n}$.

Proposition 8 Let $A_{n}$ be an $n \geq 4$ vertex antiregular graph where $n$ is an even integer. Then graph $A_{n}$ is a mean graph and for its average degree $p=\left[A_{n}\right]=n / 2$ holds. It follows that $A_{n}$ has exactly two mean vertices (exceptional vertices) with degree $\mathrm{p}=\mathrm{n} / 2$.

Proof. It is known that the edge number $m\left(A_{n}\right)$ of an n-vertex antiregular graph is $m\left(A_{n}\right)=\lceil n / 2\rceil\lfloor n / 2]$. It follows that if $\mathrm{n}$ is even integer then $\mathrm{p}=\left[\mathrm{A}_{\mathrm{n}}\right]=\mathrm{n} / 2$ and the corresponding edge number is $m\left(A_{n}\right)=n^{2} / 4$.

Remark 4 One can easily determine the degree deviation of antiregular graphs $A_{n}$ with $n \geq 4$ even vertex number. It is

$S\left(A_{n}\right)=2 \sum_{i=1}^{p\left(A_{n}\right)-1} i=2 \sum_{i=1}^{n / 2-1} i$

where $\mathrm{p}\left(\mathrm{A}_{\mathrm{n}}\right)=\mathrm{n} / 2$.

Let uv be an edge of a connected n-vertex graph G. Edge uv is called a strong edge of $\mathrm{G}$, if $\mathrm{d}(\mathrm{u})+\mathrm{d}(\mathrm{v}) \geq \mathrm{n}$, and edge $u$ is called a weak edge of $\mathrm{G}$, if $\mathrm{d}(\mathrm{u})+\mathrm{d}(\mathrm{v})$ $<\mathrm{n}$ holds. It is easy to check that in a connected antiregular graph $\mathrm{A}_{\mathrm{n}}$ every edge is strong.

The construction of pseudo-antiregular mean graphs is based on the following concept. Let $A_{n}$ be a traditional $n$-vertex connected antiregular graph with $n$ vertices where $n \geq 4$ is an arbitrary even integer and $p\left(A_{n}\right)=n / 2$. Now, by inserting $k$ novel vertices of degree $n / 2$ into $A_{n}$ as a result of this operation one obtains an $\mathrm{N}$-vertex pseudo-antiregular mean graph $\mathrm{PA}_{\mathrm{N}}(\mathrm{n}, \mathrm{k})$ with centrality parameter $\mathrm{p}(\operatorname{PA}(n, k))=n / 2$ and with vertex number $N=n+k$. As can be observed, the vertex 
sequences of graphs $\mathrm{A}_{\mathrm{n}}$ and $\mathrm{PA}_{\mathrm{N}}(\mathrm{n}, \mathrm{k})$ are different, but the degree sets of both graphs are identical.

In Fig. 6 six really mean graphs with equal centrality parameter $\mathrm{p}=3$ are depicted. Graph $\mathrm{J}_{1}$ is isomorphic to the traditional connected 6-vertex antiregular graph $\mathrm{A}_{6}$. Graphs $\mathrm{J}_{2}, \mathrm{~J}_{3}, \mathrm{JA}_{4}, \mathrm{JB}_{4}$ and $\mathrm{JC}_{4}$ are pseudo-antiregular mean graphs.

Their common properties are as follows: all of them have mean vertices and mean edges, they have identical degree sets $(1,2,3,4,5)$, and for them the corresponding degree deviation is equal, namely $\mathrm{S}=6$.
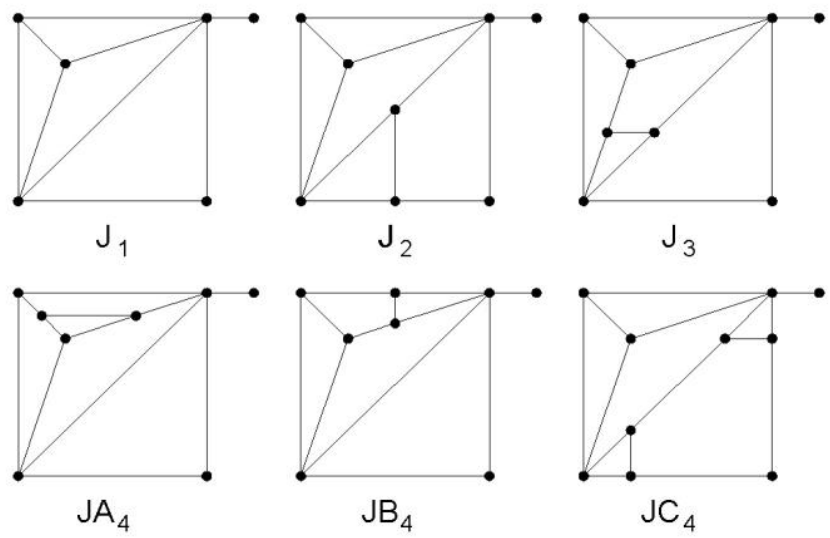

Figure 6

Non-regular graphs with the same degree set $(1,2,3,4,5)$ and the same degree deviation $S=6$

Every edge of antiregular graph $\mathrm{J}_{1}$ is a strong edge. Graphs $\mathrm{J}_{2}, \mathrm{~J}_{3}, \mathrm{JA}_{4}$ and $\mathrm{JB}_{4}$ are 8 -vertex graphs, they have 3 strong edges and 9 weak edges. Graphs $J_{2}$ and $J_{3}$ have exactly 2 and 3 mean edges, respectively. Graphs $\mathrm{JA}_{4}$ and $\mathrm{JB}_{4}$ are non-isomorphic graphs including 4 mean edges. Graph $\mathrm{JC}_{4}$ with 10 vertices has also 4 mean edges, among its 15 edges every edge is a weak edge.

From the previous considerations the following proposition yields.

Proposition 9 Let $\mathrm{n}$ and $\mathrm{k}$ be integer numbers where $\mathrm{n} \geq 4$ is even, and $\mathrm{k} \geq 0$. Then, for appropriately selected $\mathrm{n}$ and $\mathrm{k}$ parameters there exist (n-1) degreed really mean graphs with centrality parameter $\mathrm{p}=\mathrm{n} / 2$. Such graphs are the traditional antiregular graphs $A_{n}$ with even $n \geq 4$ vertex number and $k=0$, moreover the corresponding pseudo-antiregular mean graphs $\mathrm{PA}_{\mathrm{N}}(\mathrm{n}, \mathrm{k})$ with vertex number $\mathrm{N}=\mathrm{n}+\mathrm{k}$ where $\mathrm{k} \geq 1$. 


\section{Two Conjectures}

Additionally, for the structural characterization of connected mean graphs we introduce two novel graph irregularity indices formulated as

$$
\begin{aligned}
& \operatorname{IRD}(G)=\frac{2 N_{\Delta} N_{\delta}}{N_{\Delta}+N_{\delta}}(\Delta-\delta) \\
& \operatorname{IRR}(G)=\frac{n}{2}(\Delta-\delta)
\end{aligned}
$$

where $\mathrm{N}_{\Delta}$ is the number of vertices of degree $\Delta$ and $\mathrm{N}_{\delta}$ is the number of vertices of degree $\delta$, respectively.

Let $\mathrm{G}$ be an arbitrary non-regular connected $\mathrm{n}$-vertex and m-edge graph with maximum degree $\Delta$ and minimum degree $\delta \geq 1$. For graph $\mathrm{G}$, the following conjectures are established.

Conjecture 1 It is conjectured that

$S(G)=\sum_{i=1}^{n}\left|d_{i}-\frac{2 m}{n}\right| \geq \frac{2 N_{\Delta} N_{\delta}}{N_{\Delta}+N_{\delta}}(\Delta-\delta)=\operatorname{IRD}(G)$

Conjecture 2 It is conjectured that

$S(G)=\sum_{i=1}^{n}\left|d_{i}-\frac{2 m}{n}\right| \leq \frac{n}{2}(\Delta-\delta)=\operatorname{IRR}(G)$

Proposition 10 [7]: For connected bidegreed graphs $\mathrm{G}(\Delta, \delta)$ with $\mathrm{n}$ vertices and $\mathrm{m}$ edges it has been proved that

$S(G(\Delta, \delta))=\sum_{i=1}^{n}\left|d_{i}-\frac{2 m}{n}\right|=\frac{2 N_{\Delta} N_{\delta}}{N_{\Delta}+N_{\delta}}(\Delta-\delta)$

where $n=N_{\Delta}+N_{\delta}$.

Proposition 11 Let $\mathrm{G}$ be a connected bidegreed graph $\mathrm{G}(\Delta, \delta)$ with n-vertices and m-edges. Then

$$
\begin{aligned}
& S(G(\Delta, \delta))=\sum_{i=1}^{n}\left|d_{i}-\frac{2 m}{n}\right|=\frac{2 N_{\Delta} N_{\delta}}{N_{\Delta}+N_{\delta}}(\Delta-\delta)= \\
& =\operatorname{IRD}(G(\Delta, \delta)) \leq \frac{n}{2}(\Delta-\delta)=\operatorname{IRR}(G(\Delta, \delta))
\end{aligned}
$$


where equality holds if $\mathrm{G}$ is a balanced bidegreed graph, (where $\mathrm{n} \geq 4$ is an even integer, and $\mathrm{N}_{\Delta}=\mathrm{N}_{\delta}=\mathrm{n} / 2$ holds.)

Proof. Because $G$ is a bidegreed graph where $n=N_{\Delta}+N_{\delta}$, one obtains that

$S(G(\Delta, \delta))=\frac{2 N_{\Delta} N_{\delta}}{N_{\Delta}+N_{\delta}}(\Delta-\delta)=\frac{2 N_{\Delta}\left(n-N_{\Delta}\right)}{n}(\Delta-\delta)$

Consider the monotonically increasing function defined by

$g\left(N_{\Delta}\right)=\frac{2}{n} N_{\Delta}\left(n-N_{\Delta}\right)$

Its maximum value with respect to $\mathrm{N}_{\Delta}$ can be computed from

$\frac{\partial g\left(N_{\Delta}\right)}{\partial N_{\Delta}}=\frac{2}{n}\left(n-2 N_{\Delta}\right)=2-\frac{4}{n} N_{\Delta}=0$

As can be observed, function $\mathrm{g}\left(\mathrm{N}_{\Delta}\right)$ has a maximum value if $\mathrm{N}_{\Delta}=\mathrm{N}_{\delta}=\mathrm{n} / 2$ is fulfilled.

Consequently, for n-vertex connected bidegreed graphs

$S(G(\Delta, \delta)) \leq \frac{n}{2}(\Delta-\delta)=\operatorname{IRR}(G(\Delta, \delta))$,

and equality holds if $\mathrm{n} \geq 4$ is an even integer and $\mathrm{N}_{\Delta}=\mathrm{N}_{\delta}=\mathrm{n} / 2$.

Remark 5 If $\mathrm{n}$ is an odd integer, then for any n-vertex bidegreed graph $\mathrm{S}(\mathrm{G})<$ $\operatorname{IRR}(\mathrm{G})$ holds. Moreover, if $\mathrm{n}$ is even integer, but $\mathrm{N}_{\Delta} \neq \mathrm{N}_{\delta}$ for a bidegreed graph $\mathrm{G}$, then IRR(G) will always be larger than $\mathrm{S}(\mathrm{G})$. For example, if $\mathrm{G}$ is the 4-vertex star $\mathrm{K}_{1,3}$ then $\mathrm{S}\left(\mathrm{K}_{1,3}\right)=3<4=\operatorname{IRR}\left(\mathrm{K}_{1,3}\right)$.

Concerning the validity of Conjecture 1 and Conjecture 2, it can be shown that equality in formulas (6) and (7) is satisfied for a broad class of tridegreed mean graphs.

Proposition 12 There exist infinitely many n-vertex really mean tridegreed graphs $\mathrm{H}_{\mathrm{n}}$ for which $\mathrm{S}\left(\mathrm{H}_{\mathrm{n}}\right)=\operatorname{IRD}\left(\mathrm{H}_{\mathrm{n}}\right)=4$ holds.

Proof. The result follows from the properties of mean graphs $\mathrm{H}_{\mathrm{n}}$ depicted in Fig. 5 .

Proposition 13 As it is demonstrated in Fig. 4, if $n \geq 4$ is an even integer, then there exist infinitely many $n$-vertex balanced bidegreed mean graphs $F_{n}$ for which $\mathrm{S}\left(\mathrm{F}_{\mathrm{n}}\right)=\operatorname{IRD}\left(\mathrm{F}_{\mathrm{n}}\right)=\operatorname{IRR}\left(\mathrm{F}_{\mathrm{n}}\right)=\mathrm{n}$ holds.

Proof. Because bidegreed graphs $F_{n}$ are balanced mean graphs the result follows from Proposition 11.

Remark 6 Consider a connected graph $G$ with $n$ vertices and m edges. Let $A(G)$ and $L(G)$ be the corresponding adjacency and Laplacian matrices of graph $G$, 
respectively. Denote by $\lambda_{\mathrm{k}}(1 \leq \mathrm{k} \leq \mathrm{n})$ and $\mu_{\mathrm{k}}(1 \leq \mathrm{k} \leq \mathrm{n})$ the eigenvalues of matrices $A(G)$ and $L(G)$.

Because for an n-vertex and m-edge connected graph $\mathrm{G}$

$\sum_{k=1}^{n} \lambda_{k}^{2}=\sum_{k=1}^{n-1} \mu_{k}=2 m$

holds, it follows that if $G$ is a mean graph with centrality parameter $p(G)$, then

$p(G)=\frac{1}{n} \sum_{k=1}^{n} \lambda_{k}^{2}=\frac{1}{n} \sum_{k=1}^{n} \mu_{k}$

In Ref. [8] several non-isomorphic 10-vertex graphs with their Laplacian eigenvalues are presented. All of them are really mean and Laplacian equienergetic graphs having identical centrality parameter $\mathrm{p}=5$. For these mean graphs the corresponding edge number is equal to $m=n^{2} / 4=25$.

\section{Additional Considerations}

The discriminating power of various graph irregularity indices have been tested and compared in several publications [9-21]. We have seen that the degree deviation $\mathrm{S}(\mathrm{G})$ is poorly suited for discriminating among mean graphs. Its lowlevel discriminating performance was demonstrated primarily on unicyclic graphs having identical centrality parameter $\mathrm{p}=2$. This means that degree deviation measure $\mathrm{S}(\mathrm{G})$ is unable to classify (order) mean graphs according to their structural irregularity.

In what follows it will be shown that the discriminatory power of $S(G)$ is poor not only for mean graphs but for balanced bidegreed graphs as well. Starting with Proposition 11, balanced bidegreed graphs are characterized by the following property:

Proposition 14 Let $\mathrm{G}(\Delta, \delta)$ be an n-vertex and m-edge balanced bidegreed graph where $\mathrm{n} \geq 4$ is an even integer and the equality $\mathrm{N}_{\Delta}=\mathrm{N}_{\delta}=\mathrm{n} / 2$ holds. Then

$S(G(\Delta, \delta))=\sum_{i=1}^{n}\left|d_{i}-\frac{2 m}{n}\right|=\frac{n}{2}(\Delta-\delta)=\operatorname{IRR}(G(\Delta, \delta))$

Proof. Since $\mathrm{N}_{\Delta}=\mathrm{N}_{\delta}=\mathrm{n} / 2$, this implies that

$2 m=\Delta N_{\Delta}+\delta N_{\delta}=\frac{\Delta n}{2}+\frac{\delta n}{2}=\frac{n}{2}(\Delta+\delta)$ 
Consequently, one obtains

$$
\begin{gathered}
S(G(\Delta, \delta))=\sum_{i=1}^{n}\left|d_{i}-\frac{2 m}{n}\right|=N_{\Delta}\left|\Delta-\frac{\Delta+\delta}{2}\right|+N_{\delta}\left|\delta-\frac{\Delta+\delta}{2}\right| \\
=\frac{n}{2}(\Delta-\delta)
\end{gathered}
$$

There are infinitely many balanced bidegreed graphs characterized with the above property. Such graphs can be generated by the so-called partial edge-subdivision operation (PES transformation) performed on $\mathrm{R} \geq 3$ regular graphs. By using PES transformation on the $R \geq 3$ regular graph $G_{R}$ with $n_{R}$-vertices we can insert $n / 2$ new vertices of degree $\delta=2$ into the parent graph $\mathrm{G}_{\mathrm{R}}$. As a result of this transformation, one obtains a balanced bidegreed graph $\mathrm{G}(\mathrm{R}, 2)$ with vertex number $\mathrm{n}(\mathrm{G}(\mathrm{R}, 2))=2 \mathrm{n}_{\mathrm{R}}$ and edge number $\mathrm{m}(\mathrm{G}(\mathrm{R}, 2))=\mathrm{n}_{\mathrm{R}}(\mathrm{R}+2) / 2$.

Example 2 The concept of PES transformation is illustrated by graphs depicted in Fig. 7. The 3-regular graph $\mathrm{G}_{\mathrm{T}}$ is the graph of the 6-vertex trigonal prism. Balanced bidegreed graphs $G_{j}(j=1,2,3)$ constructed from $G_{T}$ are 12-vertex nonisomorphic graphs containing 6 vertices of degree 3 and 6 vertices of degree 2 .

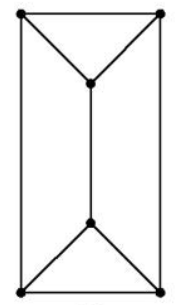

$\mathrm{G}_{\mathrm{T}}$

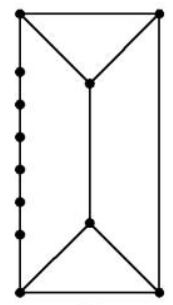

$\mathrm{G}_{1}$

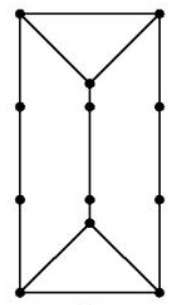

$\mathrm{G}_{2}$

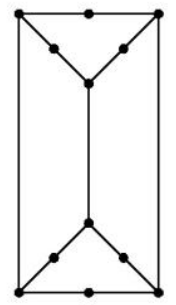

$\mathrm{G}_{3}$

Figure 7

Balanced bidegreed graphs constructed from the 3-regular graph $\mathrm{G}_{\mathrm{T}}$

Non-isomorphic balanced bidegreed graphs $G_{j}(j=1,2,3)$ do not belong to the family of mean graphs, however, they have identical degree deviation given by

$S\left(G_{j}\right)=\sum_{i=1}^{12}\left|d_{i}-\frac{5}{2}\right|=\operatorname{IRD}\left(G_{j}\right)=\operatorname{IRR}\left(G_{j}\right)=6$

As can be observed, balanced bidegreed graphs depicted in Fig. 7 cannot be discriminated by irregularity indices $\mathrm{S}(\mathrm{G})$, IRD $(\mathrm{G})$ and $\operatorname{IRR}(\mathrm{G})$.

It is interesting to note that the so-called Albertson irregularity index [9] defined by 


$$
A L(G)=\sum_{u v \in E}|d(u)-d(v)|
$$

seems to be more efficient for graph irregularity characterization, i.e. it possesses a better discriminatory performance. Computing the Albertson indices for graphs $\mathrm{Gj}(\mathrm{j}=1,2,3)$, we have $\mathrm{AL}\left(\mathrm{G}_{1}\right)=2, \mathrm{AL}\left(\mathrm{G}_{2}\right)=6$ and $\mathrm{AL}\left(\mathrm{G}_{3}\right)=12$.

Pokoradi demonstrated in [23] that the comparative evaluation of the discriminating power of irregularity indices is problematic in many cases.

For example, consider balanced graphs $\mathrm{J}_{1}, \mathrm{JA}_{4}$ and $\mathrm{JB}_{4}$ depicted in Fig. 6 . For their irregularity indices one obtains: $\mathrm{S}\left(\mathrm{J}_{1}\right)=\mathrm{S}\left(\mathrm{JA}_{4}\right)=\mathrm{S}\left(\mathrm{JB}_{4}\right)=6$, and $\mathrm{AL}\left(\mathrm{J}_{1}\right)=\mathrm{AL}\left(\mathrm{JA}_{4}\right)=$ $\operatorname{AL}\left(\mathrm{JB}_{4}\right)=16$.

Denote by $M_{1}$ the first Zagreb index of a connected graph with $n$ vertices and $m$ edges $[17,19]$. It is interesting to note that the irregularity index defined by

$I R M_{1}(G)=M_{1}(G)-\frac{4 m^{2}}{n}=\sum_{i=1}^{n}\left(d_{i}-\frac{2 m}{n}\right)^{2}$

has an equivalent discriminating performance with degree deviation $S(G)$ if $G$ is a mean graph. This observation is based on the following considerations: If connected graphs $\mathrm{G}$ and $\mathrm{H}$ are mean graphs with identical centrality parameter $\mathrm{p}$, and the only difference between the degree sequences of $\mathrm{G}$ and $\mathrm{H}$ is that number $\mathrm{N}_{\mathrm{p}}$ of vertices of degree $\mathrm{p}$ is different, then $\operatorname{IRM}_{1}(\mathrm{G})=\operatorname{IRM}_{1}(\mathrm{H})$ holds.

We end our study by pointing out a recently published paper [22] containing new results on extremal graphs having maximal degree deviation. Ghalavand and Ashrafi proved [22] that among all n-vertex connected graphs the maximal degree deviation is attained for a particular set of complete split graphs. An n-vertex complete split graph denoted by $\mathrm{Cs}(\mathrm{n}, \mathrm{k})$ is a connected bidegreed graph consisting of an independent set of $n-k$ vertices and a clique of $k$ vertices, such that each vertex of the independent set is adjacent to each vertex of the clique [24]. According to Ref. [22] the corresponding degree deviation can be calculated as

$S(C s(n, k))=\frac{2 k}{n}(n-k)(n-k-1)$.

Among n-vertex connected graphs the maximal degree deviation belongs to the complete split graphs $\mathrm{Cs}(\mathrm{n}, \mathrm{k})$ listed below, where $\mathrm{k}$ is defined as follows

- $\mathrm{k}=\mathrm{n} / 3$, if $\mathrm{n}$ is divisible by 3 ,

- $\mathrm{k}=(\mathrm{n}-1) / 3$, if $\mathrm{n}-1$ is divisible by 3 ,

- $\mathrm{k}=(\mathrm{n}-2) / 3$ if $\mathrm{n}-2$ is divisible by 3 or

$-\mathrm{k}=(\mathrm{n}+1) / 3$ if $\mathrm{n}+1$ is divisible by 3 . 
From the previous considerations, we can conclude that among 12-vertex connected graphs the maximal degree deviation belongs to the complete split graph $\operatorname{Cs}(n=12, k=4)$. In this case, the corresponding degree deviation is

$S(C s(12,4))=\frac{2 \cdot 4}{12}(12-4)(12-4-1)=\frac{112}{3}=37.333$

As can be observed, this degree deviation is considerably larger than that of balanced bidegreed graphs depicted in Fig. 7 .

\section{Acknowledgements}

The authors would like to thank Dr. László Horváth for his extensive help with computer graphics.

\section{References}

[1] C. Godsil, G. Royle, Algebraic Graph Theory, Springer-Verlag, New York, 2001

[2] V. Nikiforov, Eigenvalues and degree deviation in graphs, Linear Algebra Appl., 414 (2006) 347-360

[3] N. V. R. Mahadev, U. N. Peled, Threshold Graphs and related Topics, in: Annals of Discrete Mathematics, Vol. 57, Elsevier Science B. V., Amsterdam, 2004

[4] L. Nebeskỳ, On connected graphs containing exactly two points of the same degree, Časopis, Pést. Mat, 98 (1973) 305-306

[5] A. Ali, A survey of antiregular graphs, Contrib. Math. 1 (2020) 67-79

[6] B. Bollobás, Modern Graph Theory, Graduate Texts in Mathematics, Springer Verlag, New York, 1998

[7] T. Réti, I. Milovanović, E. Milovanović, M. Matejić, On graph irregularity indices with particular regard to degree deviation, paper under publication in FILOMAT

[8] D. Stevanović, Large sets of noncospectral graphs with equal Laplacian energy, MATCH Commun. Math. Comput. Chem. 61 (2009) 463-470

[9] M. O. Albertson, The irregularity of a graph, Ars Comb. 46 (1997) 219-225

[10] A. T. Balaban, Highly discriminating distance-based topological index, Chem. Phys. Lett. 89 (1982) 399-404

[11] I. Gutman, On Discriminativity of Vertex-Degree-Based Indices, Iranian Journal of Mathematical Chemistry, 3, (2012) 95-101

[12] T. Došlić, On Discriminativity of Zagreb Indices, Iranian Journal of Mathematical Chemistry, 3 (2012) 25-34 
[13] T. Došlić, T. Reti, Novel degree-based molecular descriptors with increased discriminating power, Acta Polytech. Hung. 9 (2012) 17-30

[14] I. Gutman, Topological indices and irregularities measures, Bull. Inter. Math. Virt. Inst. 8 (2018) 469-475

[15] B. Furtula, I. Gutman, M. Dehmer, On structure-sensitivity of degree-based topological indices, Appl. Math. Comput. 219 (2013) 8973-8978

[16] T. Reti, D. Dimitrov, On Irregularity of Bidegreed Graphs, Acta Polytech. Hung. 10 (2013) 117-134

[17] A. Hamzeh, T. Reti, An Analogue of Zagreb Index Inequality Obtained from Graph Irregularity Measures, MATCH Commun. Math. Comput. Chem.72 (2014) 669-683

[18] I. Gutman, Irregularity of molecular graphs, Kragujevac J. Sci. 38 (2016) $71-81$

[19] T. Reti, E. Tóth-Laufer, On the construction and comparison of graph irregularity indices, Kragujevac J. Sci. 39 (2017) 53-75

[20] M. Matejić, B. Mitić, E. Milovanović, I. Milovanović, On Albertson irregularity measure of graphs, Sci. Publ. State Univ. Novi Pazar Ser. A: Apple. Math. Inform. Mech. 11 (2019) 97-106

[21] A. Ali, E. Milovanović, M. Matejić, I. Milovanović, On the upper bounds for the degree-deviation of graphs, J. Appl. Math. Comp. 62 (2020) 179187

[22] A. Ghalavand, A. R. Ashrafi, On a conjecture about degree deviation measure of graphs, Trans. Comb. 1 (2021) 1-8

[23] L. Pokoradi, Graph model-based analysis of technical systems, IOP Conf. Series: Materials Science and Engineering 393 (2018) 012007, doi: 10.1088/1757-899X/393/1/012007

[24] D. Stevanović, Spectral Radius of Graphs, Academic Press, Amsterdam, 2015 